\title{
Child- and adult-orientated breakfast cereals: a cross sectional analysis of nutrient profile
}

\author{
B. Barlow and T.J. Butler \\ Department of Clinical Sciences and Nutrition, University of Chester, Chester, CH1 4BJ, UK.
}

Data from the most recent UK National Child Measurement Programme (NCMP) shows the prevalence of childhood obesity to be $10 \%$ of children in reception year (aged 4-5 years) and $20 \%$ in year 6 (aged 10-11 years) ${ }^{(1)}$. The most recent National Diet and Nutrition survey ${ }^{(2)}$ identified cereal and cereal products as the main source of free sugars in children aged 1.5-3 years and 4-10 years. Whilst breakfast cereals (BCs) are fortified with vitamins and minerals, there is growing concern over their sugar content and the link with weight gain and obesity, and cardiovascular risk in both children and adults ${ }^{(3)}$. Using a cross-sectional approach we aimed to explore differences in nutrient content and recommended portion sizes between adult- and child-orientated BCs available in the UK.

BCs were chosen from four main supermarkets in the UK. BCs were classified as either adult- or child-orientated using a validated approach $^{(4)}$ that considered if the $\mathrm{BC}$ packaging contained a licensed character, television, or movie theme, any other cartoon drawing, or an activity or promotion that was directed at children. Nutrient information was taken from details provided by the manufacturer. All data were analysed using IBM SPSS (Version 24.0).

One hundred and thirty five $(n=135)$ cereals were identified, with $10(7.4 \%)$ classified as children's BC and $125(92.6 \%)$ classified as adult BC. No significant differences for energy content per $100 \mathrm{~g}$ were observed between groups (table 1). Carbohydrate, sugars, protein and salt were all present at significantly greater quantities in the child-oriented $\mathrm{BC}$ when compared with the adult cereal group (table 1). Total fat $(\mathrm{g} / 100 \mathrm{~g})$ content did not differ significantly between adult and child $\mathrm{BC}$ groups $(8.8 \pm 8.7 \mathrm{~g}$ vs. $6.6 \pm 6.3 \mathrm{~g}$, respectively). Fibre content $(\mathrm{g} / 100 \mathrm{~g})$ was significantly $(P<0.05)$ lower in child $\mathrm{BC}$ when compared to adult $\mathrm{BC}(6 \cdot 0 \pm 2 \cdot 9 \mathrm{~g}$ vs. $8 \cdot 3 \pm 3 \cdot 2 \mathrm{~g}$, respectively).

Table 1. Energy and nutrient content of cereals analysed

\begin{tabular}{|c|c|c|c|c|c|c|c|c|c|c|c|c|}
\hline \multirow{2}{*}{ Group } & \multicolumn{2}{|c|}{ Portion size $(\mathrm{g})$} & \multicolumn{2}{|c|}{ Energy (kcal/100 g) } & \multicolumn{2}{|c|}{$\begin{array}{c}\text { Carbohydrate } \\
(\mathrm{g} / 100 \mathrm{~g})\end{array}$} & \multicolumn{2}{|c|}{ Sugars $(g / 100 \mathrm{~g})$} & \multicolumn{2}{|c|}{ Protein $(\mathrm{g} / 100 \mathrm{~g})$} & \multicolumn{2}{|c|}{$\begin{array}{c}\text { Salt } \\
(\mathrm{g} / 100 \mathrm{~g})\end{array}$} \\
\hline & Mean & $\overline{\mathrm{SD}}$ & Mean & $\overline{\mathrm{SD}}$ & Mean & $\overline{\mathrm{SD}}$ & Mean & $\overline{\mathrm{SD}}$ & Mean & $\overline{\mathrm{SD}}$ & Mean & $\mathrm{SD}$ \\
\hline Adult BC $(\mathrm{n}=125)$ & $40 \cdot 6$ & $5 \cdot 4$ & $392 \cdot 5$ & $40 \cdot 6$ & $66 \cdot 2$ & $6 \cdot 8$ & $17 \cdot 3$ & $7 \cdot 5$ & $10 \cdot 4$ & $6 \cdot 3$ & $0 \cdot 3$ & 0.3 \\
\hline Child BC $(n=10)$ & $32 \cdot 5 *$ & $5 \cdot 0$ & $397 \cdot 2$ & $38 \cdot 8$ & $73 \cdot 5 *$ & $8 \cdot 6$ & $23 \cdot 1 *$ & $7 \cdot 8$ & $8 \cdot 0^{*}$ & $2 \cdot 1$ & $0 \cdot 6^{*}$ & $0 \cdot 4$ \\
\hline
\end{tabular}

$\mathrm{BC}$, breakfast cereal. Mean portion size, carbohydrate, sugar, protein and salt were significantly different when compared to adult BCs (Mann-Whitney U Test): $* P<0.05$ vs. adult group.

These results demonstrate that children's BC in the UK contains significantly greater amounts of sugar and salt than adult BC, and is consistent with established literature from other countries ${ }^{(5)}$. The contribution of $\mathrm{BC}$ to sugar intake is an important health target and may be of use when considering strategies to improve energy balance in this group.

1. NHS Digital (2018) Statistics on Obesity, Physical Activity and Diet. England: 2018 [online] Available at https://digital.nhs.uk/catalogue/PUB30258 [Accessed 8 April, 2018].

2. NDNS (2012) National Diet and Nutrition Survey: headline results from Years 1,2 and 3 combined (2008/9-2010/11) [online] Available at https:// www.gov.uk/government/uploads/system/uploads/attachment_data/file/207707/ndns-y3-executive-summary_final.pdf [Accessed 8 April, 2018].

3. Vos MB, Kaar JL, Welsh JA et al. (2017) Circ 135, e1017-34.

4. Schwartz MB, Vartanian LR, Wharton CM et al. (2008) J Am Diet Assoc 108, 702-5.

5. Monique PK, Cameron C \& Philippe S (2017) Health Promot Chronic Dis Prev Can 37, 266-73. 